\title{
Custom Reverse Total Shoulder Arthroplasty
}

\author{
Brian T Grisez ${ }^{1}$, Mark A Goodman ${ }^{2}$ and Brock A Lindsey ${ }^{1 *}$
}

${ }^{1}$ Department of Orthopaedics, West Virginia University, Morgantown, WV, USA

${ }^{2}$ Department of Orthopaedic Surgery, University of Pittsburgh Medical Center, Pittsburgh, PA, USA

\begin{abstract}
Reverse total shoulder arthroplasty (rTSA) initially faltered because of glenoid component failure. Modern design utilizes a large glenosphere component and relies upon a functional deltoid for arm elevation. We report novel use of rTSA to revise a chronically dislocating TSA. The patient underwent proximal humeral resection for chondrosarcoma, requiring sacrifice of the deltoid and all proximal insertions including the latissimus dorsi. Twoyears post-operatively, he had good stability, no pain, and was using his arm more than he had in years. rTSA is a salvage option for failed TSA, even with absent deltoid function and lack of a latissimus.
\end{abstract}

Keywords: Reverse total shoulder arthroplasty; Proximal humeral neoplasm; Shoulder arthroplasty dislocation

\section{Introduction}

Reverse total shoulder arthroplasty (rTSA) has gained significant popularity in the past decade. Although introduced in the 1970s, it initially failed to gain popularity because of unacceptable failure rates of the glenoid component. However, the modern rTSA design by Grammont, which utilizes a large glenosphere component, has become the treatment of choice for severe cuff-tear arthropathy and is the only indication approved by the Food and Drug Administration (FDA) $[1,2]$. Because of its success, its uses have been expanded to treat other shoulder pathology including proximal humeral neoplasms, nonunion fractures, and also as a salvage procedure for failed conventional arthroplasty [3-6]. We report a novel use of the reverse total shoulder arthroplasty in a patient who underwent multiple revisions of an unconventional arthroplasty with a constrained proximal humeral component. His initial indication for replacement was a proximal humeral resection for a bony neoplasm, which required sacrifice of the deltoid muscle and removal of latissimus insertion. Because the design relies upon a functional deltoid to permit arm elevation, it is typically contraindicated when there is severe neurologic impairment or absence of the muscle [1].

\section{Case Report}

A 63-year-old man presented in 2004 for an elective rotator cuff repair of his right shoulder. About this time, he began to have pain in his left shoulder which was worse at night and often awakened him from sleep. Examination did not reveal any specific pathology; he had full range of motion (ROM) and exceptional strength in the entire arm, including the shoulder. A radiograph of the arm revealed a mass suspicious for a bony neoplasm. In January 2005, the patient underwent limb-sparing surgery requiring removal of the proximal half of his humerus, as well as the majority of the deltoid muscle. A hemiarthroplasty was also performed to salvage function of the arm. The patient had a revision in February 2008 because of chronic dislocation of his hemiarthroplasty; the hemiarthroplasty was converted to a total shoulder arthroplasty with a constrained proximal humeral component. Unfortunately, the patient had recurrent anterior dislocations (Figure 1), limited ROM in the joint, and pain. On examination, the implant could be palpated just deep to the skin. Three treatment options were considered: 1) nonoperative management; 2) complete explant of all hardware, which would leave him with a flail shoulder; and 3) replacing the constrained implant with a custom reverse prosthesis. After extensive discussion and collaboration with Biomet, Inc. (Warsaw, IN), a custom reverse total shoulder prosthesis was designed. The patient underwent surgery in August 2012 (Figure 2); his hospital course was unremarkable. At the 10-week follow-up, he reported some burning pain over his lateral shoulder at about the level of the prosthetic stem-humeral junction, which radiated into his forearm. It was felt that his pain was secondary to a brachial plexus traction neuropathy due to a lengthening of the arm with the new prosthetic components and lack of deltoid for active reduction of his shoulder joint. The patient was started on Pregabalin $75 \mathrm{mg}$ three times a day and given a sling to use when performing physical activity like walking. At his two-year follow-up, he was using his left arm more than he had in years. His active ROM of his left shoulder included $20^{\circ}$ of abduction, $5^{\circ}$ of forward flexion, and $30^{\circ}$ of

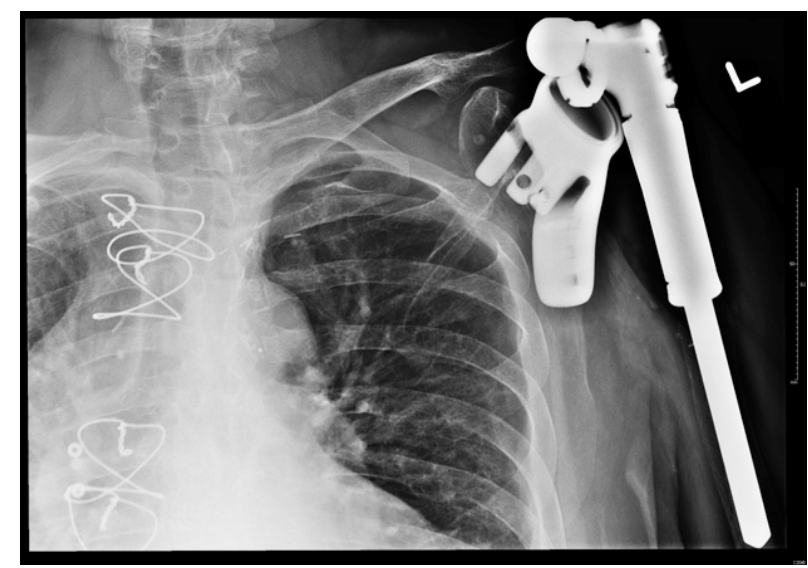

Figure 1: The anterior-posterior $(\mathrm{AP})$ radiograph identifies a custom constrainedtype total shoulder arthroplasty with evidence of dislocation and superior anterior displacement of the humeral component.

${ }^{*}$ Corresponding author: Brock A Lindsey, Department of Orthopaedics, West Virginia University, PO Box 9196, Morgantown, WV 265069196, USA, Tel: 3042931317; Fax: 3042937070; E-mail: blindsey@hsc.wvu.edu

Received April 25, 2017; Accepted May 23, 2017; Published May 25, 2017

Citation: Grisez BT, Goodman MA, Lindsey BA (2017) Custom Reverse Total Shoulder Arthroplasty. J Cancer Sci Ther 9: 430-432. doi: 10.4172/19485956.1000454

Copyright: ( $) 2017$ Grisez BT, et al. This is an open-access article distributed under the terms of the Creative Commons Attribution License, which permits unrestricted use, distribution, and reproduction in any medium, provided the original author and source are credited. 
extension (Figures 3 and 4). Range of motion of his left elbow included full extension and $150^{\circ}$ of flexion with normal hand function. He had had no symptoms of brachial plexopathy, was off all medication, and reported no episodes of instability or pain.

\section{Discussion}

Although severe impairment of deltoid function is a relative contraindication to rTSA, we present a case where such an implant was used in a patient who had no deltoid muscle or active latissimus. Currently accepted indications for rTSA include rotator cuff tear arthopathy (CTA), proximal humeral neoplasms, non-union fractures, and as revision procedure for failed traditional replacement or hemiarthroplasty [3-6].

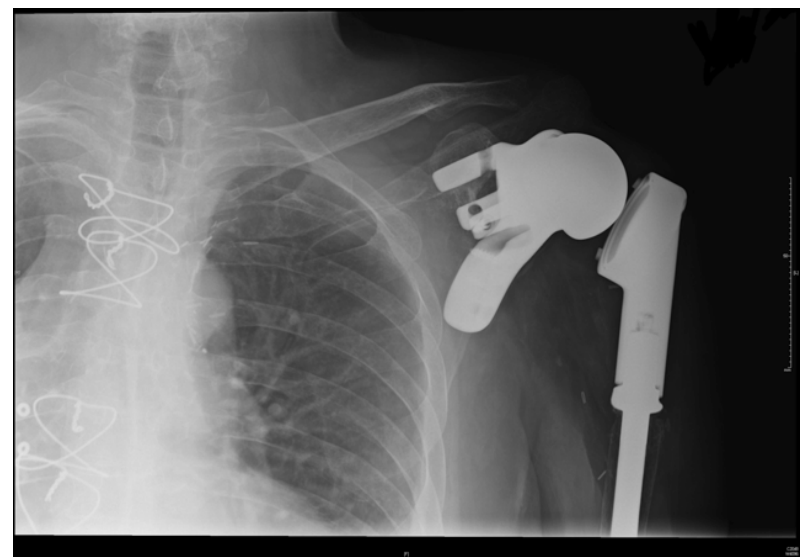

Figure 2: The post-operative AP radiograph demonstrates replacement of the constrained-type arthroplasty with a custom total reverse style arthroplasty. Note the glenoid and stem components were preserved from the previous surgery.

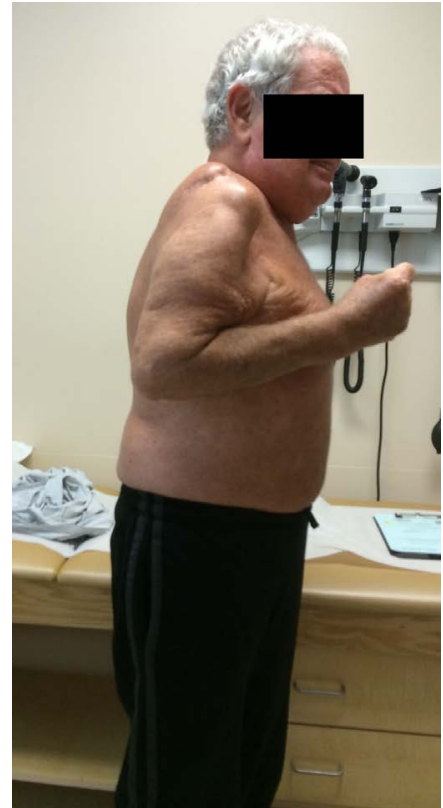

Figure 3: Patient demonstrating 110 degrees of elbow flexion at two year postoperative follow-up. Having use of the forearm allowed him to perform most activities of daily living independently.

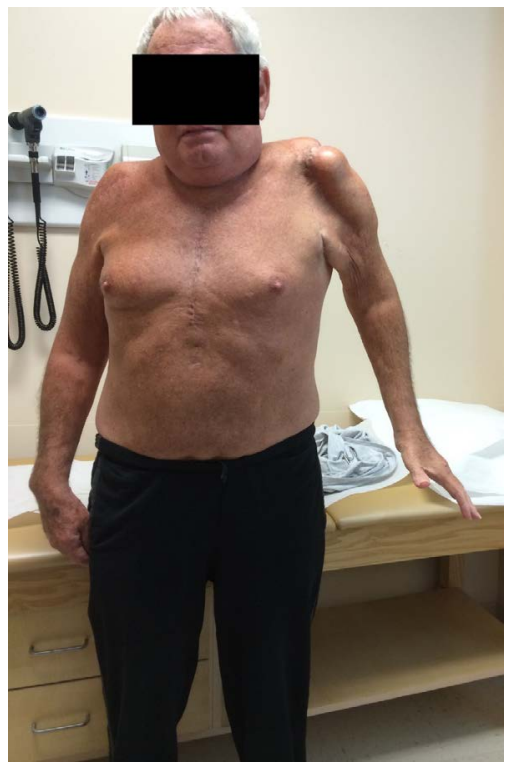

Figure 4: Patient at maximum abduction after custom reverse total shoulder with no deltoid or remaining shoulder gridle.

A completely deficient deltoid is thought to be a strong contraindication to rTSA, specifically if dysfunction includes the anterior head. A cadaveric study has demonstrated that balanced abduction is severely disrupted if both segments of the anterior deltoid are impaired [7]. However, another cadaver study by Gulotta concluded anterior deltoid deficiency can be compensated by both the subscapularis and middle deltoid during elevation to $60^{\circ}$ [8]. Seemingly, both the anterior and middle deltoid contribute to function in rTSA, although the anterior head is responsible for a larger burden of the function. Groh described a series of 36 patients with deltoid deficiency after various shoulder operations, of which 25 had loss of the anterior or anterior and middle heads; 28 reported fair or poor shoulder function as determined by activities of daily living [9]. Tay reported on one case of rTSA for CTA and concurrent irreparable middle deltoid avulsion with good outcome [10]. Schneeberger performed rTSA on 19 patients who failed deltoid flap reconstruction for irreparable rotator cuff tears. These patients all had a substantial defect in the anterolateral portion of their deltoid from the flap reconstruction surgery. All had objective improvement as measured by the Constant score and all were either very satisfied $(15 / 19)$ or satisfied (4/19) with the result [11]. Thus, partial deltoid impairment, even in the anterior head, is not an absolute contraindication to rTSA [12].

Latissimus dorsi (LD) transfer, as described by Itoh, is an option to improve arm elevation and abduction in those patients with deltoid deficiency [13]. The procedure involves detaching the LD from its insertion on the humerus and dividing the muscle about the level of the serratus anterior. The pedicle is then inverted and tunneled posterior to the pectoralis major and the LD insertion tendon is sutured to the insertion tendon of the deltoid. The LD origin fibers are then sutured to the anterior and lateral acromion or costal origin of the trapezius. Goel reported one case of massive cuff tear arthropathy and deltoid avulsion treated with rTSA and LD transfer; after one year, the patient had $135^{\circ}$ of forward elevation, $20^{\circ}$ of external rotation, and internal rotation to L2 [14]. This procedure may have been an option for our patient; however, his LD insertion had been lost as a consequence of his prior 
surgeries. To our knowledge, there is no other literature describing use of rTSA and concurrent repair of a deficient deltoid.

The reported complication rate after rTSA varies from $19 \%$ to $68 \%$ and the most frequent complications include neuropathy, infection, scapular notching, dislocation, fractures, and baseplate failure [15]. Post-operative neuropathy, as seen in our patient, may be the result of intraoperative traction or manipulation, retractor placement, or lengthening of the arm secondary to the humeral stem implant $[15,16]$.

Clinically significant neurologic injury has been reported in $1 \%$ to $4 \%$ of all patients undergoing rTSA [17]. Inherent to the operative technique, the humerus is externally rotated, abducted, and retracted posteriorly to allow for appropriate glenoid exposure, which may place stress on the brachial plexus resulting in neuropathy [1]. Revision may be an additional factor causing neurologic injury compared to primary rTSA because of dissection in distorted anatomy and difficulty with component removal. Interestingly, neuropathic pain developed as a delayed complication, rather than immediately in the post-operative period. This complication was likely due to arm lengthening during activity secondary to unopposed forces of gravity but was not apparent early on because of the use of the shoulder immobilizer.

\section{Conclusion}

The goal of surgery was to relieve pain, improve elbow/hand function, and prevent dislocation. Active abduction and elevation were severely limited but the patient reported more use of his arm without instability compared to before surgery. An rTSA should be considered for salvage even without any functional deltoid or latissimus function.

\section{References}

1. Boileau P, Watkinson DJ, Hatzidakis AM, Balg F (2005) Grammont reverse prosthesis: Design, rationale, and biomechanics. J Shoulder Elbow Surg 14: 147S-61S.

2. Drake GN, O'Connor DP, Edwards TB (2010) Indications for reverse total shoulder arthroplasty in rotator cuff disease. Clin Orthop Relat Res 468: 15261533.

3. De Wilde L, Sys G, Julien Y, Van Ovost E, Poffyn B, et al. (2003) The reversed delta shoulder prosthesis in reconstruction of the proximal humerus after tumour resection. Acta Orthop Belg 69: 495-500
4. Kaa AK, Jorgensen PH, Sojbjerg JO, Johannsen HV (2013) Reverse shoulde replacement after resection of the proximal humerus for bone tumours. Bone Joint J 95: 1551-1555.

5. Levy J, Frankle M, Mighell M, Pupello D (2007) The use of the reverse shoulder prosthesis for the treatment of failed hemiarthroplasty for proximal humeral fracture. J Bone Joint Surg Am 89: 292-300.

6. Wall B, Nove-Josserand L, O'Connor DP, Edwards TB, Walch G (2007) Reverse total shoulder arthroplasty: A review of results according to etiology. J Bone Joint Surg Am 89: 1476-1485.

7. Schwartz DG, Kang SH, Lynch TS, Edwards S, Nuber G, et al. (2013) The anterior deltoid's importance in reverse shoulder arthroplasty: A cadaveric biomechanical study. J Shoulder Elbow Surg 22: 357-364.

8. Gulotta LV, Choi D, Marinello P, Wright T, Cordasco FA, et al. (2012) Anterio deltoid deficiency in reverse total shoulder replacement: A biomechanical study with cadavers. J Bone Joint Surg Br 94: 1666-1669.

9. Groh GI, Simoni M, Rolla P, Rockwood CA (1994) Loss of the deltoid after shoulder operations: An operative disaster. J Shoulder Elbow Surg 3: 243-253.

10. Tay AK, Collin P (2011) Irreparable spontaneous deltoid rupture in rotator cuff arthropathy: The use of a reverse total shoulder replacement. J Shoulder Elbow Surg 20: e5-8.

11. Schneeberger AG, Muller TM, Steens W, Thur C (2014) Reverse total shoulder arthroplasty after failed deltoid flap reconstruction. Arch Orthop Trauma Surg 134: 317-323.

12. Ladermann A, Walch G, Denard PJ (2013) Reverse shoulder arthroplasty in patients with pre-operative impairment of the deltoid muscle. Bone Joint $\mathrm{J} 95$ 1106-1113.

13. Itoh Y, Sasaki T, Ishiguro T, Uchinishi K, Yabe $Y$ et al. (1987) Transfer of latissimus dorsi to replace a paralysed anterior deltoid. A new technique using an inverted pedicled graft. J Bone Joint Surg Br 69: 647-651.

14. Goel DP, Ross DC, Drosdowech DS (2012) Rotator cuff tear arthropathy and deltoid avulsion treated with reverse total shoulder arthroplasty and latissimus dorsi transfer: Case report and review of the literature. J Shoulder Elbow Surg 21: e1-e7.

15. Cheung E, Willis M, Walker M, Clark R, Frankle MA (2011) Complications in reverse total shoulder arthroplasty. J Am Acad Orthop Surg 19: 439-449.

16. Ladermann A, Lubbeke A, Melis B, Stern R, Christofilopoulos P, et al. (2011) Prevalence of neurologic lesions after total shoulder arthroplasty. J Bone Joint Surg Am 93: 1288-1293.

17. Walch G, Bacle G, Ladermann A, Nove-Josserand L, Smithers CJ (2012) Do the indications, results, and complications of reverse shoulder arthroplasty change with surgeon's experience? J Shoulder Elbow Sur 21: 1470-1407. 